American Journal of Immunology 7 (1): 9-11, 2011

ISSN 1553-619X

(C) 2011 Science Publications

\title{
An Exceptional Case Report of Primary Cutaneous Cryptococcosis in Human Immunodeficiency Virus
}

\author{
${ }^{1,2}$ A.S. Valan, ${ }^{2}$ S. Kumar, ${ }^{1}$ K. Ramalingam, ${ }^{1}$ J. Suria, \\ ${ }^{1} \mathrm{G}$. Manoharan and ${ }^{2} \mathrm{C}$. Chandrasekar \\ ${ }^{1}$ Department of HIV Fellowship, \\ International Training and Education Centre for Health, Chennai, India \\ ${ }^{2}$ Department of HIV Fellowship, Government Hospital of Thoracic Medicine, \\ Tambaram, Chennai, India
}

\begin{abstract}
Problem statement: Cryptococcosis is an infection caused by the encapsulated yeast Cryptococcus neoformans, a dimorphic fungus. In patients with AIDS, skin manifestations represent the second most common site of disseminated Cryptococcosis. Approach: Mr. A 42 year old farmer was referred from Voluntary Counseling and Testing Centre (VCTC) with positive HIV status to the outpatient department with one week history of asymptomatic discrete papular lesions over the face. Extirpation of the papule led to expression of the colorless watery material, which on Indian ink and Grams staining showed presence of Cryptococci and his CD4 count was found to be 58 cells cu. $\mathrm{mm}^{-1}$. Sabourauds Dextrose Agar (SDA) culture of the material from the skin lesion revealed growth of Cryptococci. CSF, blood and sputum culture were negative for pathogens, Chest X-ray did not reveal any abnormal findings. Results: A diagnosis of primary Cutaneous Cryptococcosis was made and the patient was treated with Intravenous Amphotericin B followed by oral Fluconazole along with other supportive measures and Anti retroviral therapy. He showed remarkable improvement following therapy. Conclusion: In advanced stages of Immunodeficiency, patients may present only with umbilicated papules without any associated constitutional symptoms. High degree of clinical suspicion, thorough history, detailed clinical examination and appropriate laboratory investigations are needed to diagnose primary cutaneous cryptococcosis as these lesions may be misdiagnosed as Molluscum Contagiosum.
\end{abstract}

Key words: Encapsulated yeast, umbilicated papule, sabouraud dextrose agar, amphotericin B

\section{INTRODUCTION}

Cryptococcosis is an infectious disease that usually affects the respiratory tract and subsequently disseminates to other systems such as central nervous system and urinary tract (Cohen et al., 2010). In HIV-infected patients, cryptococcosis is typically seen when the CD4 count is less than 50100 cells $\mu \mathrm{L}^{-1}$ and is an AIDS-defining illness (National AIDS Control Organization, 2007). Approximately $15 \%$ of the patients with systemic dissemination of Cryptococcosis have secondary involvement of the skin (Dharmshale et al., 2006) but primary Cutaneous Cryptococcosis is a rare manifestation in HIV.

\section{MATERIALS AND METHODS}

Mr. A is a 42 year old Farmer with positive HIV status, presented with multiple, umbilicated, nodular and papular lesions over inner and outer canthi of eyes, eyelids and para nasal areas (Fig. 1). Extirpation of the papule led to expression of the colorless watery material. On gram stain showed (Fig. 2) budding yeasts and on Indian ink showed presence of Cryptococci. This watery material from the papules inoculated and cultured in Sabouraud Dextrose Agar (SDA) after 4 days grew white-creamy and shiny colonies of Cryptococci at room temperature (Fig. 3). But microscopy and culture of blood, Cerebro spinal fluid and sputum did not yield any Cryptococcal organism. His CD4 count was 58 cells cu.mm ${ }^{-1}$. Other essential investigations done were within normal limits.

Corresponding Author: A.S. Valan, Department of HIV Fellowship, International Training and Education Centre for Health, Chennai, India Tel: +91-9952502250 Fax- 91-44-22414200 
Am. J. Immunol., 7 (1): 9-11, 2011

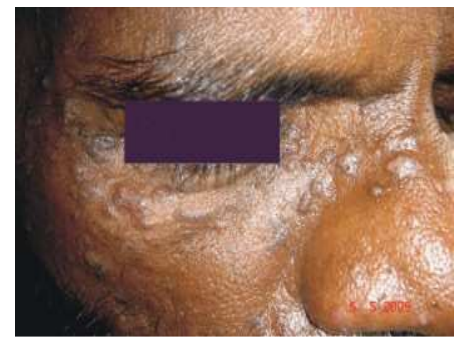

Fig. 1: Cutaneous Cryptococcal lesions of face

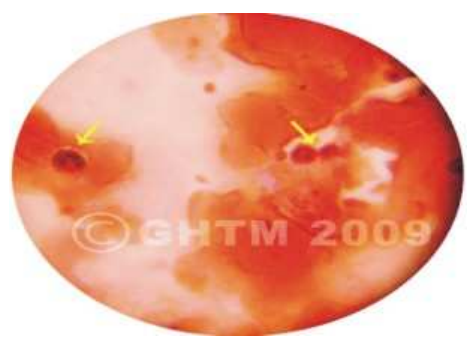

Fig. 2: Budding yeast Cells in Gram Stain

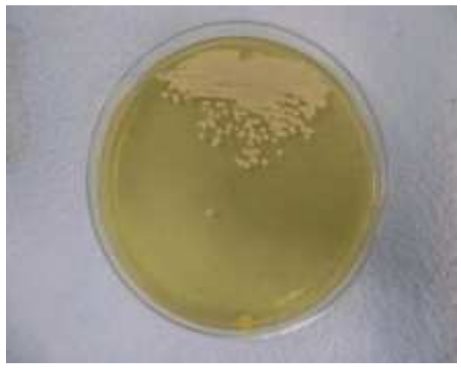

Fig. 3: White-Creamy mucoid Cryptococal Colonies in SDA

\section{RESULTS}

Based on the History and Clinical examination supported by the laboratory investigation a diagnosis of cutaneous cryptococcosis was made. He was treated with inj. Amphotericin B $40 \mathrm{mg}\left(0.75 \mathrm{mg} \mathrm{Kg}^{-1}\right.$ body weight/Day) Once daily Intravenous Slow infusion) for 14 days followed by Tab. Fluconazole $400 \mathrm{mg}$. Cutaneous lesions in this patient healed within 7 days of systemic anti fungal Amphotericin-B (Basu et al., 2010; Jill and Richard, 2002; Baginksi and Czumb, 2009). Subsequently he was initiated on Anti retroviral therapy (Zidovudine, Lamivudine and Efavirenz) after 14 days (National AIDS Control Organization, 2007) of Inj. Amphotericin B. Consolidation therapy of Fluconazole $400 \mathrm{mg}$ q.d $\left(5-\mathrm{mg} \mathrm{kg}^{-1} \mathrm{dose}^{-1}\right)$ orally for 8 weeks followed by maintenance therapy of Fluconazole $200 \mathrm{mg}$ (3-6mg/kg/day) once daily per os was administered after Intravenous Amphotericin B as per national Opportunistic Infection guidelines 2007.

\section{DISCUSSION}

Recognition of primary Cutaneous Cryptococcosis as a clinical entity has long been debated. The lesions vary greatly in morphology and mimic other dermatologic entities (Blauvelt and Kerdel, 1992) like Molluscum contagiosum (Ramdial et al., 2008) Penicillium marnefii, Histoplasmosis, Kaposi Sarcoma. An altered immunological status is an important factor for developing this disease. There is often a clear history of trauma or exposure to soil or birds preceding the development of the lesion (Saag et al., 2000). The lesion is confined to the skin without systemic involvement.

In this patient there were no associated symptoms with his cutaneous lesions and Gram stain, Indian ink stain and SDA culture were negative for Cryptococcal organism thus Disseminated Cryptococcosis was ruled out. He is asymptomatic now and his CD4 cell count rose to 216 cells cu.mm after 6 months of being on ART. It was planned to continue secondary prophylaxis of Fluconazole till next CD4 cell count after 6 months as recommended by the national Guidelines.

Studies from affluent settings show that in the era of HAART, the causes of mortality in people infected with HIV are not all due to AIDS-related events (Bonnet et al., 2002; 2005). However, in resource poor settings, opportunistic infections still contribute to a large number of AIDS related deaths and this may be accelerated in those not taking prophylaxis and those unable to access medical care (Teja et al., 2007).

HIV infected patients are at greater risk of acquiring Cryptococcal disease. In advanced immunodeficiency stage as the disease progresses (Durden and Elewski, 1994), Cryptococci commonly manifests as meningitis and then progress to disseminated form. Rarely Cryptococci manifests as primary cutaneous infection (Thomas and Schwartz, 2001). Early diagnosis and treatment of cutaneous cryptococcal infection will prevent dissemination of the organism into systemic infection and would increase the survival of immune compromised individuals (Rajagopalan et al., 2009).

\section{CONCLUSION}

Cutaneous Cryptococcal infection presenting with umbilicated papules could be misdiagnosed as Molluscum contagiosum, Penicillium marnefii, Histoplasmosis. Proper diagnosis and timely initiation 
of HAART has a significant role in improving the survival of immunocompromised individuals diagnosed with Cryptococcal infection.

\section{REFERENCES}

Basu, S., D. Chakraborty and S. Das, 2010. Susceptibility of Candida species isolated from HIV infected and newborn candidaemia patients to amphotericin B. OnLine J. Biol. Sci., 10: 109-113. DOI: 10.3844/ojbsci.2010.109.113

Blauvelt, A. and F.A. Kerdel, 1992. Cutaneous cryptococcosis mimicking Kaposi's sarcoma as the initial manifestation of disseminated disease. Int. J. Dermatol., 31: 279-280. PMID: 1634295

Bonnet, F., C. Lewden, T. May, L. Heripret and E. Jougla et al., 2005. Opportunistic infections as causes of death in HIV-infected patients in the HAART era in France. Scand J. Infect. Dis., 37: 482-487. PMID: 16089023

Bonnet, F., P. Morlat, G. Chene, P. Mercie and D. Neau et al., 2002. Causes of death among HIV-infected patients in the era of highly active antiretroviral therapy, Bordeaux, France, 1998-1999. HIV. Med., 3: 195-199. PMID: 12139658

Baginksi, M. and J. Czumb, 2009. Amphotericin B and its new derivatives-mode of action. Curr. Drug Metab. 10: 459-469. PMID: 19689243

Cohen, J., S.M. Opal and W.G. Powderly, 2010. Infectious Diseases. 3rd Edn., Mosby/Elsevier, USA., ISBN: 0323045790, pp: 1918.

Dharmshale, S.N., S.A. Patil, A. Gohil, A. Chowdhary and C. Oberoi, 2006. Disseminated crytococcosis with extensive cutaneous involvement in AIDS. Indian J. Med. Microbiol., 24: 228-230. PMID: 16912448

Durden, F.M. and B. Elewski, 1994. Cutaneous involvement with Cryptococcus neoformans in AIDS. J. Am. Acad. Dermatol., 30: 844-848. PMID: 8169258
Jill, A.M. and T. Richard, 2002. AmBisome: Liposomal formulation, structure, mechanism of action and pre-clinical experience. J. Antimicrob. Chemother., 49: 21-30. DOI: 10.1093/jac/49.suppl_1.21

National AIDS Control Organization, 2007. Antiretroviral therapy guidelines for HIV-infected adults and adolescents including post-exposure prophylaxis. 1st Edn., National AIDS Control Organisation, Ministry of Health and Family Welfare, India, pp: 126.

Rajagopalan, N., J.B. Suchitra, A. Shet, Z.K. Khan and J. Wigdahl et al., 2009. Mortality among HIVinfected patients in resource limited settings: A case controlled analysis of inpatients at a community care center. Am. J. Infect. Dis., 5: 219224. DOI: 10.3844/ajidsp.2009.219.224

Ramdial, P.K., E. Calonje, Y. Sing, N.A. Chotey and J. Aboobaker, 2008. Molluscum-like cutaneous cryptococcosis: A histopathological and pathogenetic appraisal. J. Cutan. Pathol., 35: 10071013. PMID: 18537859

Saag, M.S., R.J. Graybill, R.A. Larsen, P.G. Pappas and J.R. Perfect et al., 2000. Practice guidelines for the management of cryptococcal disease. Infectious Diseases Society of America. Clin. Infect. Dis., 30: 710-718. PMID: 10770733

Teja, V.D., T. Sudha and V. Lakshmi, 2007. Causes and pattern of mortality in HIV-infected, hospitalized patients in a tertiary care hospital: A fourteen year study. Indian J. Med. Sci., 61: 555561. PMID: 17932446

Thomas, I. and R.A. Schwartz, 2001. Cutaneous manifestations of systemic cryptococcosis in immunosupressed patients. J. Med., 32: 259-266. PMID: 11958272 